\title{
From Multicultural Agents to Culture-Aware Robots
}

\author{
Matthias Rehm \\ Faculty of Engineering and Science \\ Aalborg University \\ 9200 Aalborg, Denmark \\ matthias@create.aau.dk
}

\begin{abstract}
In our work on developing multicultural agents we have primarily relied on the analysis of video recordings of multimodal face to face interactions between humans, where the videos have been collected in different cultures. This posed some questions concering the cultural biases of the analysis due to the cultural background of the annotators. For the development of culture-aware robots we have now adopted a strategy that takes this cultural bias into account as a feature of the development process by integrating the potential user groups from different cultures into this process. We exemplify this approach with a case study on affective body movements for a humanoid robot.
\end{abstract}

\section{Introduction}

In previous research we showed how a cross-cultural multimodal corpus of human face to face interactions can serve as empirical foundation for implementing multicultural agents (e.g. [13]). To this end, video recordings have been annotated in relation to a number of communicative phenomena like gestures, postures, proxemics, communication management, etc. Based on these annotations, statistical models have been derived and implemented in embodied conversational agents in order to simulate culture-specific communicative behavior in these agents. Although this was a successful approach, there have always been some discomforts about it due to the following reasons:

- Subjectivity of the annotation: Although there are measures that can be taken to ensure a certain degree of objectivity in the annotation of the video data (e.g. ensuring high inter-rater agreement by calculating kappa values, restricting free text annotations, training of annotators), manual annotations will always contain a certain degree of subjective interpretation of the phenomena that are under observation. This subjective interpretation may become a liability if the number of annotators is limited (which is often the case due to costs), meaning that single subjective interpretations get more weight in the models derived from the annotations.

- Cultural bias of the annotation: Our cultural background influences to a large degree how we behave in and how we interpret situations involving social

M. Kurosu (Ed.): Human-Computer Interaction, Part I, HCII 2013, LNCS 8004, pp. 431-440, 2013.

(C) Springer-Verlag Berlin Heidelberg 2013 
interactions. Thus, apart from being subjective, annotations of behavior will be (unconsciously) biased by the cultural background of the annotator because what they deem "normal" in social interactions will be structured by those unconscious shared heuristics from the cultural group they belong to.

- Cultural bias of the design and the implementation of the agents: The same unconscious cultural bias might also influence the design and implementation of the agents. Which kinds of gestures look "normal" and how gestures are performed have for instance been shown to vary between cultures, and thus cultural heuristics are likely to influence how movements and behaviors of an agent are realized on the surface.

Meanwhile, we have moved to physical agents, i.e. robots. Here the difference between humans and robots is more apparent, due e.g. to limited expressive channels or reduced degrees of freedom. For instance the Nao platform which we are using has less joints in arms and legs then a human, making movements look different, independent on how careful movements have been designed.

Thus, we suggest a new methodological approach for modeling the behavior of robots and for making them culture-aware, which could also be of benefit for the use in multicultural agents. Instead of collecting data from human interactions, potential users of the system have to create the behavior themselves, allowing us to collect a cross-cultural database of behavioral parameters for the robotic system under development. This reduces subjective or cultural biases that were previously introduced. Instead, the cross-cultural database of behavioral parameters allows data-mining the statistical models that will drive the generation of communicative behavior in the robot based on informants that are immersed in the target culture.

In this paper, we are first looking at cultural influences on the developement and evaluation process (Section 2), before we propose our method of co-creation which is loosely based on ideas from classical participatory design in HCI (Section 3). Then we report on the results of our co-creation experiment for affective body movements (Section 4) before we discuss how this method might resolve some of the discomforts discussed above (Section (5). The paper ends with a short conclusion (Section 6).

\section{Related Work}

In recent years, culture has been identified as an important variable that influences not only the development of interactive systems, but more fundamentally also experimental work that often serves as an empirical basis for interactive systems like virtual agents or robotic companions. Henrich and colleagues [7] present a thought provoking analysis of experimental work in psychology covering an analysis of the top journals in Psychology over four years (2003-2007) that shows that general conclusions about human psychology are pre-dominantly based on a very specific sample which is only representative for small fraction of society. They called this the WEIRD sample for Western, Educated, Industrialized, Rich, and Democratic, and highlighted that $68 \%$ of all samples were from 
the US, and overall $96 \%$ from Western industrialzed societies, although WEIRD societies represent only $12 \%$ of the world population. To put it bluntly, current claims about general human psychology e.g. concerning cooperation, moral reasoning or visual perception, are based solely on Western university and college students, making these universal claims seem at least be very questionable.

Following this argument, Blanchard [1] examined research in the field of educational systems and analyzed contributions to the two main conference in the area (ITS and AIED) over the last decade (2002-2010) focusing on the origin of first authors as well as samples used in evaluations and shows that there is a similar WEIRD tendency to be found in this area of research.

Earlier, Elfenbein and Ambady [5] showed the importance of taking participants cultural background into account as one variable in experimental settings when they investigated in-group advantages for the production and recognition of emotional facial expressions. In earlier work, we have taken these suggestions into account for a cross-cultural examination of the interpretation of emotional facial expressions in virtual agents [8] that also showed an in-group effect concerning the designers' as well as the observers' cultural group. Moreover, we have presented an in-depth analysis of the challenges of developing multicultural agents focusing on the problem of implicit biases by the developers' and the users' cultural backgrounds [11.

\section{Users as Co-creators}

The method we are proposing here is inspired by "classical" HCI work on participatory design (e.g. [3]; 2]). This design paradigm claims that it is necessary to involve users early on in the development process to ensure an optimal solution in terms of usability and features of a product. The PD process allows potential users (as well as stakeholders) bringing their perspective into the development process. In recent work we have shown that especially in cross-cultural developments it is an absolute requirement to involve the target group and let them become co-designers of the envisioned system [14.

Here we report on our adoption of the approach for the development of cultureaware robots. In our case study, we focus on affective body movements for humanoid robots. Instead of analyzing culture-specific human face to face interactions like we did in previous work on culture aware agents, we allow users to design affective body movements for the robots directly by manipulating a set of well defined movement parameters. Result of this co-creation experiment is a cross-cultural data base that can be used to derive a probabilistic model to drive the behavior of the robot.

\subsection{Affective Body Movements in Humanoid Robots}

A particular challenge with robots is their restricted possibilities regarding expressive behaviors, which convey important information in human interactions. In embodied conversational agents for instance, facial expressions are prominent 
means for conveying affective information (e.g. [15]). But most humanoid robots so far are extremely limited in terms of facial expressions. In our case study, we worked with the Nao, a humanoid robot that allows no facial movements. Thus, affective information can best be displayed by bodily movements or robot specific means like sound or light. One set of parameters that is repeatedly mentioned in related work on affective body movements for robots is based on Laban's effort and shape dimensions [9]. Two recent examples highlight this use of Laban's movement analysis. Masuda and Kato [10] show that observers are able to distinguish between four different emotions when a basic movement is modified on the basis of Laban's parameters. Takahashi and colleagues [16] report on the design of emotional body movements based on Laban's parameters for a robot resembling a teddy bear. Despite its low expressivity (raising/lowering arms, moving arms to front of body, tilt/shake head), recognition rates for three emotions (joy, fear, sadness) are acceptable.

\subsection{Experimental Design}

The co-creation experiment is based on Laban's effort and shape dimensions 9]. Thus, by manipulating parameters on these dimensions, participants can create body movements that (from their perspective) exhibit affective information. The goal of this co-creation experiment is to locate the value ranges for the different dimensions that relate to the expression of emotions for several cultures. Following work by Gross and colleagues [6], the experiment concentrates on one single movement that has to be manipulated to make it affectively expressive.

So far the database consists of around 600 entries across 4 different cultures (Danish, German, Japanese, Greek) plus additional entries for other cultures that have not yet reached a critical mass. Participants demographics show that roughly a third are female $(35 \%)$ and two thirds male $(65 \%)$ with an age range from 20 to 45 (mean: 28.5, SD: 6.44). Most participants have no hands-on experience with robots.

Data collection was organized as a web experiment, where participants were randomly presented with the task of adding an affective connotation to a standard movement from the robot (knocking at a door). This follows the ideas presented by Gross and colleagues on isolating relevant parameters by focusing on a single movement 6]. Participants were presented with six tasks corresponding to the following six basic emotions [4]: anger, disgust, fear, happiness, sadness, and surprise. To create affective motions, participants could manipulate the Laban parameters weight, time, and space. Because the parameter names time, weight and space have been shown in a pilot test to be not readily understandable by participants, they were changed to speed, power and path for the experiment, where speed (time) denotes how fast the movement is performed, power (weight) denotes how much strength is put into the movement, and path (space) denotes if the motion is direct or indirect.

Figure 1 gives an impression of the web interface. Each parameter could be changed on a three point scale: speed (slow to fast), power (weak to strong), path (direct to indirect). For each movement, participants were then asked to 


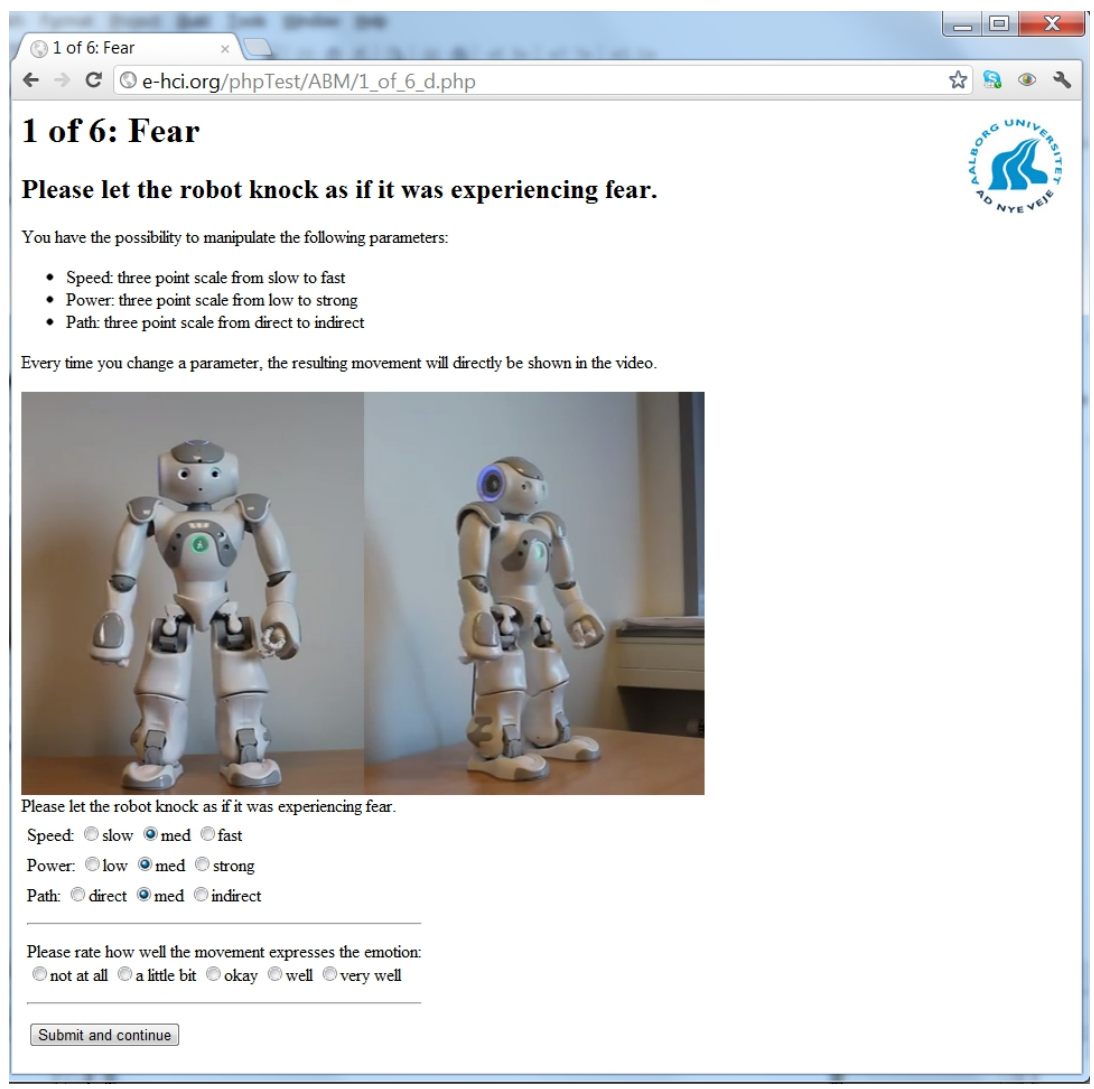

Fig. 1. Interface for the web experiment. Tasks are presented in random order.

indicate their satisfaction with the movement on a five point Likert scale. Each parameter modification is directly visible in the video window, which shows the robot's movement according to the current parameter values showing the robot from two perspectives (front view and side view) allowing for a better insight into how parameter modifications change the movement.

\section{From Co-creation to Culture-Aware Behavior}

Here we report on a comparison of German and Japanese data. More detailed results including Greek and Danish data can be found in [12].

Looking at the cross-cultural database, several significant differences are visible in the parameter values for some of the emotions. Figure 2 provides an overview of these differences for the two exemplary cultures German and Japanese. The results highlight that we find significant differences for all parameters, but not necessarily for all or even for the same emotions. For instance, speed of the gesture (time parameter) is important for happiness but not for any 

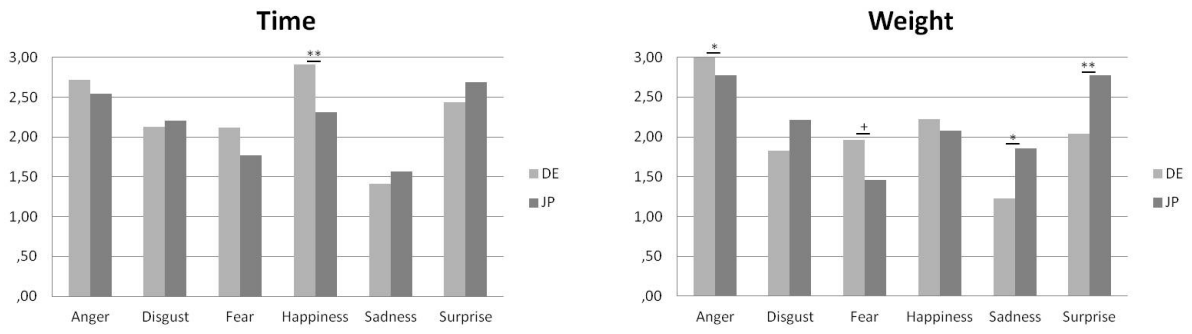

Space

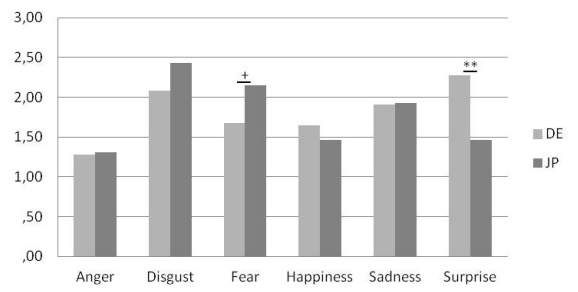

Fig. 2. Average values for time, weight and space parameter for the six emotions across two exemplary cultures (German, Japanese). Significance levels: $+: \mathrm{p}<0.1 ;{ }^{*}: \mathrm{p}<0.05$; **: $\mathrm{p}<0.01$.
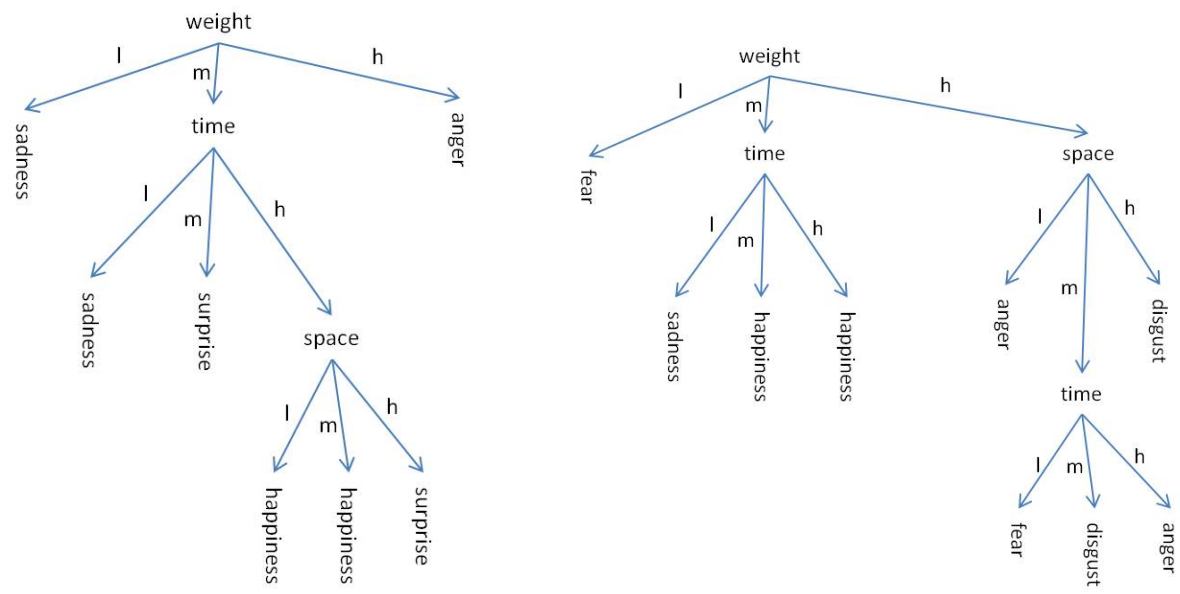

Fig. 3. Decisions trees (J48 pruned) for the two example cultures German and Japanese

of the other emotions, while the power of a gesture (weight parameter) effects the connotation of anger, fear, sadness, and suprise.

Data mining techniques are employed on the cross-cultural database collected by the web experiment. Decision trees allow for deciding for the relative importance of the different parameters in relation to the emotional content of the movement. Coming back to Figure 2 we see that by the significance of the differences between the cultures that the weight put into the movement seems to 


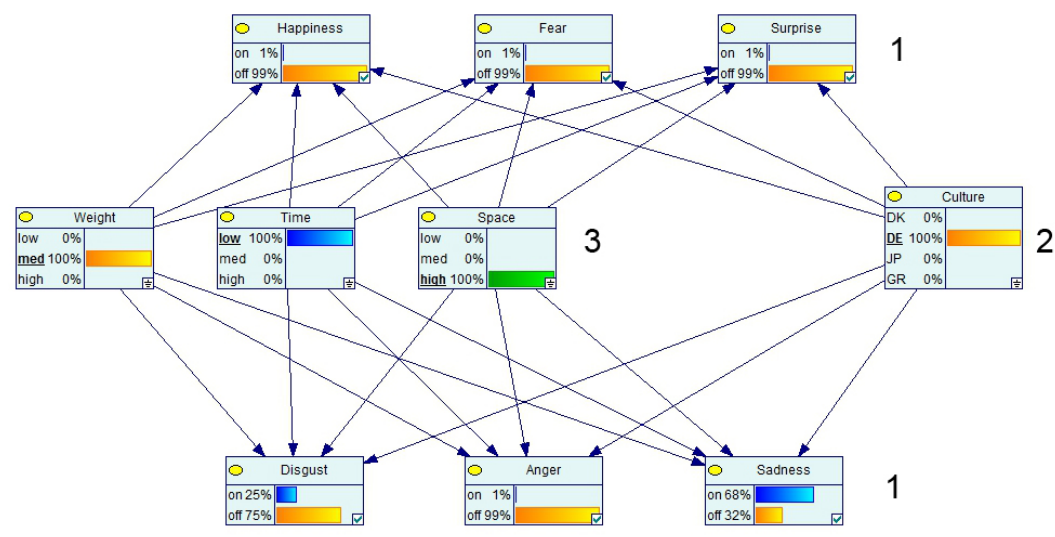

Fig. 4. Bayesian network modeling the relation between emotions (1), cultural background (2), and Laban parameters (3)

be differentiating the impression of fear as well as sadness. Figure 3 shows the two decision trees (J48) calculated on the German and Japanese data. What is apparent from the figure is that weight indeed is an important feature being at the root of both trees. Moreover, a cultural difference in attributing emotional connotation to a movement is apparent from the culture-specific decision trees. Low weight is attributed to sadness for the German sample independent of the values of the other parameters, whereas it is attributed to fear for the Japanese sample, again indepedent of the values of the other two parameters. Several other differences as well as similarties can be seen by comparing the two trees.

Calculating the decision trees allows for analyzing the relative importance of feature value combinations for the attribution of emotions to movements. Based on this information, a Bayesian network is build up (see Figure 4), which allows for reflecting the unreliability and ambiguity that is a trademark of phenomena like culture and emotions. It is used for generating movement patterns (Figure 4. 3.) based on the input information concerning the cultural background of the user (Figure 4, 2.) and the emotion that is to be conveyed by the body movement (Figure 4, 1.).

This allows us now to generate culture-specific affective body movements for the robot grounded in the unconscious heuristics of members of the respective cultures about affective body movements on the one hand but also of suitable body movements for robots (as compared to humans) on the other hand. Next step is to test the result with observers from the corresponding cultures.

\section{Discussion}

The motivation for exploring the the co-creation method were some discomforts we highlighted in the introduction regarding the empirical basis of our behavior 
models. In this section, we revisit these discomforts and discuss to what extent the method of co-creation addresses these discomforts.

\subsection{Subjectivity of the Annotation of Multimodal Data}

The subjectivity of the impression of affective movements from the single participant's still remains in the co-creation experiment. But using the participants as as co-creators on a large scale becomes possible by the proposed web experiment should take care of relativizing subjective interpretations. This would be difficult to achieve with standard annotations of video data due to the high effort needed on the side of the annotators.

\subsection{Cultural Bias of the Annotation of Multimodal Data}

In the co-creation experiment, this cultural bias was explicitely turned into a feature of the data collection by establishing a cross-cultural data base of movement parameters. Thus, instead of presenting a problem for the data analysis, the data collection is designed to be based on cultural bias allowing for exploring this effect for the interpretation of affective body movements. Compared to the annotation of cross-cultural video data, two layers of complexity are removed, because in case of the video data, there will be cultural bias in creating the videos (which is the target of the analysis) and there will be cultural bias in analyzing the videos by the annotators (which can be seen as unwanted noise).

\subsection{Cultural Bias for the Design and the Implementation of Agents}

Again by the method of co-creation, this cultural bias is turned into a feature of the development process, allowing for creating and comparing the culturespecific relevance of different movement parameters for the emotional connotation of movements. Thus, what the participants with a given cultural background deem as "normal" for executing an emotional gesture will be captured in the co-creation process and is generalized by the calculated models for movement generation.

Summing up, we can state that the approach presented in this paper has proven to be a promising direction for developing culture-aware robots by taking cultural biases of the intended user groups into account through a participatory development process.

\section{Conclusion}

In this paper we presented an approach that allows us to tackle the challenge of implicit cultural biases in the development of culture aware technology exemplified by affective body movements for humanoid robots. We claim that it is necessary to make these cultural biases explicit when developing multicultural applications and take them into account on several levels during the development 
process. Here we presented a co-creation experiment that allowed for collecting a cross-cultural database of movement parameters serving as a basis for deriving culture-specific behaviors for the robot. Future work includes validating the resulting behavior, again taking culture into account, this time as an experimental variable.

Acknowledgements. Special thanks go to Prof. Yukiko Nakano (Seikei University, Japan), Prof. Tomoko Koda (Osaka Institute of Technology, Japan), PD Dr. Katharina Rohlfing (Center of Excellence for Cognitive Interaction Technology, Germany), Amaryllis Raouzaiou (National Technical University of Athens, Greece), and Markus Häring (Augsburg University, Germany) for their recruiting efforts for the case study described in this paper.

\section{References}

1. Blanchard, E.: On the WEIRD Nature of ITS/AIED Conferences: A 10 Year Longitudinal Study Analyzing Potential Cultural Biases, pp. 280-285. Springer (2012)

2. Bødker, S., Iversen, O.S.: Staging a professional participatory design practice: moving pd beyond the initial fascination of user involvement. In: Proceedings of the Second Nordic Conference on Human-Computer Interaction, pp. 11-18. ACM, New York (2002)

3. Dearden, A., Rizvi, H.: Participatory design and participatory development: a comparative review. In: Proceedings of the PDC, pp. 81-91 (2008)

4. Ekman, P.: Basic emotions. In: Dalgleish, T., Power, M. (eds.) Handbook of Cognition and Emotion, ch. 3, pp. 45-60. John Wiley and Sons Ltd., Chichester (1999)

5. Elfenbein, H.A., Ambady, N.A.: When Familiarity Breeds Accuracy: Cultural Exposure and Facial Emotion Recognition. Journal of Personality and Social Psychology $85(2), 276-290(2003)$

6. Melissa Gross, M., Crane, E.A., Fredrickson, B.L.: Methodology for assessing bodily expression of emotion. Journal of Nonverbal Behavior 34, 223-248 (2010)

7. Henrich, J., Heine, S.J., Norenzayan, A.: The weirdest people in the world? Behavioral and Brain Sciences 33, 61-135 (2010)

8. Koda, T., Ishida, T., Rehm, M., André, E.: Avatar Culture: Cross-Cultural Evaluations of Avatar Facial ExpressionsAvatar Culture: Cross-Cultural Evaluations of Avatar Facial Expressions. AI \& Society, Special Issue on Enculturating HCI 24(3), 237-250 (2009)

9. Laban, R.: The mastery of movement. Dance Books (2011)

10. Masuda, M., Kato, S.: Motion rendering system for emotion expression of human form robots based on laban movement analysis. In: Proceedings of the 19th IEEE International Symposium on Robot and Human Interactive Communication, pp. 324-329 (2010)

11. Rehm, M.: Developing Enculturated Agents - Pitfalls and Strategies. In: Blanchard, E.G., Allard, D. (eds.) Handbook of Research on Culturally-Aware Information Technology. IGI Global (2010)

12. Rehm, M.: Experimental designs for cross-cultural interactions: A case study on affective body movements for HRI, pp. 78-83. IEEE Computer Society Press (2012) 
13. Rehm, M., Nakano, Y., André, E., Nishida, T., Bee, N., Endrass, B., Wissner, M., Lipi, A.A., Huang, H.-H.: From Observation to Simulation - Generating Culture Specific Behavior for Interactive Systems. AI \& Society 24, 267-280 (2009)

14. Rodil, K., Winschiers-Theophilus, H., Jensen, K.L., Rehm, M.: Homestead Creator, pp. 627-630. ACM (2012)

15. Schröder, M., Bevacqua, E., Cowie, R., Eyben, F., Gunes, H., Heylen, D., ter Maat, M., McKeown, G., Pammi, S., Pantic, M., Pelachaud, C., Schuller, B., de Sevin, E., Valstar, M., Wöllmer, M.: Building Autonomous Sensitive Artificial Listeners. IEEE Transactions on Affecite Computing 3(2), 165-183 (2012)

16. Takahashi, K., Hosokawa, M., Hashimoto, M.: Remarks on designing of emotional movement for simple communication robot. In: Proceedings of the IEEE International Conference on Industrial Technology (ICIT), pp. 585-590 (2010) 